\title{
Utilizaçáo da CIF em pacientes com sequelas de AVC
}

\author{
The use of ICFH in stroke patients
}

\author{
Ana Irene Costa de Oliveira ${ }^{1}$ Katyana Rocha Mendes da Silveira ${ }^{2}$
}

\section{RESUMO}

Objetivo. O estudo consistiu em avaliar as funções e restrições de pacientes com sequela de AVC utilizando como ferramenta a CIF, incentivar seu uso e mostrar que pode permitir uma nova visão das condiçóes de saúde. Método. Foram avaliados cinco pacientes de ambos os gêneros com quadro de hemiparesia, que se encontravam em atendimento fisioterapêutico na Clínica Escola de Fisioterapia da Universidade São Francisco (USF). Foram utilizadas ficha de avaliação neurológica, Escala de Qualidade de Vida Específica para AVE (EQVE-AVE) e a Canadian Occupational Performance Measure (COPM) para mensurar os aspectos referentes aos componentes da CIF. Resultados. Todos os pacientes apresentaram déficits de funcionalidade e restriçóes em suas Atividades de Vida Diária (AVD’s), todos de forma diferente devido aos fatores pessoais e ambientais, levando-os a um comprometimento psicossocial. Conclusáo. A CIF é uma importante ferramenta que pode avaliar o paciente com AVC, pois observa o paciente de uma forma global (funcionalidade), sabendo que fatores pessoais e ambientais estáo diretamente relacionados com a forma com que se apresenta na vida. Por ser uma ferramenta que tem visão ampla do paciente é importante para a prática clínica, pois oferece vários artifícios que melhoram a avaliação e auxilia na elaboração de um programa de tratamento individualizado.

Unitermos. CIF, AVC, Funcionalidade.

Citação. Oliveira AIC, Silveira KRM. Utilização da CIF em pacientes com sequelas de AVC.

\begin{abstract}
Objective. The aim of the study was to assess function and restriction of patients with sequel of stroke, using the International Classification of Functioning (ICF), to stimulate its use, demonstrating that this tool can provides a new way to understand the condition of patient's health. Method. Five patients of both genders were appraised with hemiparesis. They were selected from the Physiotherapy Facility, a school clinic University of Sao Francisco (USF). The dates used for the study was neurological evaluation sheet; Scale of Quality of Life specific for stroke patients; and the Canadian Occupational Performance Measure (COPM) to evaluate the aspects at the components of ICF. Results. All patients presented functioning deficit and restrictions on their Activities of Daily Living (ADL). This fact leads to a psychosocial consequence like isolation of their leisure activities or from their previous occupation. Conclusion. This study concluded that ICF is an important tool to evaluate the patients with AVC, classifying the patient not as their illness but according with their functioning. Considering that ICF has a complete vision of patients, offering several forms that improve the evaluation and support to create a program of centered treatment.
\end{abstract}

Keywords. ICF, CVA, Functioning.

Citation. Oliveira AIC, Silveira KRM. The use of ICFH in stroke patients.
Trabalho realizado na Universidade Sáo Francisco, Bragança Paulista-SP, Brasil.

1. Fisioterapeuta, graduada na Universidade São Francisco, Bragança PaulistaSP, Brasil.

2. Fisioterapeuta, graduada da Universidade do Sagrado Coração, São Paulo, SP, pós -graduada (latu sensu) pela Universidade Federal de São Paulo e (strictu sensu) pela Universidade Bandeirante de São Paulo.
Endereço para correspondência: Ana Irene C Oliveira

Universidade Sáo Francisco (USF) Rua Clarice Funck Ninni, 259 Jardim Recreio 


\section{INTRODUÇÃO}

Historicamente as intervençóes em reabilitação têm sido guiadas pelo modelo médico. Esse modelo define saúde como a ausência de doenças e, por conseguinte focaliza a avaliação e o tratamento nos sinais e nos sintomas da patologia considerando apenas o nível físico. $\mathrm{Na}$ atualidade os modelos de reabilitação refletem uma mudança de paradigma e definem saúde em termos mais amplos, indicando que fatores sociais, psicológicos e ambientais contribuem para a saúde e a qualidade de vida ${ }^{1}$.

Visando responder às necessidades de se conhecer mais sobre as doenças, em maio de 2001 após várias versões e numerosos testes, a Assembléia Mundial da Saúde aprovou a International Classification of Funtioning Disability and Health (ICF). A tradução foi realizada pelo Centro Colaborador da Organização Mundial de Saúde para a Família de Classificações Internacionais em Língua Portuguesa, com o título de Classificação Internacional de Funcionalidade, Incapacidade e Saúde (CIF)².

A CIF evoluiu a partir de uma classificação de "consequências de doenças" para uma classificação de "componentes da saúde", no qual "componentes da saúde" define o que constitui a saúde, enquanto "consequências" focaliza o impacto das doenças ou outras condiçóes que se seguem como um resultado. Neste caminho a CIF toma um lugar neutro com respeito à etiologia e permite aos pesquisadores chegarem a inferências causais com métodos científicos ${ }^{3}$.

A CIF e o Código Internacional de Doenças Décima Revisão (CID-10) são complementares, pois a CID-10 fornece um "diagnóstico" de doenças, distúrbios ou outras condições de saúde, e essas informações são complementadas pelos dados adicionais fornecidos pela CIF sobre funcionalidade. Em conjunto as informaçóes sobre o diagnóstico e sobre a funcionalidade fornecem uma imagem mais ampla e mais significativa da saúde das pessoas ${ }^{4}$.

O modelo proposto pela CIF baseia-se na junção dos modelos médico e social, e uma abordagem biopsicossocial é usada para se obter uma integração das várias dimensóes da saúde (biológica, individual e social). A funcionalidade e a incapacidade humanas são concebidas como uma interação dinâmica entre as condições de saúde (doença, trauma, lesóes, distúrbios) e os fatores contextuais (incluindo fatores pessoais e ambientais) 5 .
A CIF é uma classificação com múltiplas finalidades elaborada para servir a várias disciplinas e setores diferentes. Ela cobre todos os componentes possíveis da saúde e alguns componentes relacionados à saúde (tais como educação, trabalho, etc) para descrição e avaliação ${ }^{3,6}$.

A CIF tem duas partes, cada uma com dois componentes: A parte 1- Funcionalidade e Incapacidade que é subdividida em (a) Funções do Corpo e Estruturas do Corpo e (b) Atividades e Participação, e parte 2 - Fatores Contextuais, subdividida em (c) Fatores Ambientais e (d) Fatores Pessoais. Cada componente pode ser expresso em termos positivos e negativos e cada um contém vários domínios e em cada domínio há várias categorias, que são as unidades de classificação ${ }^{6}$.

A adoção do modelo de funcionalidade e incapacidade humana possibilita ao fisioterapeuta, em seus procedimentos de avaliação e de intervenção, considerar um perfil funcional específico para cada indivíduo. O que está de acordo com os estudos que relatam que a estrutura e o conteúdo da CIF são capazes de auxiliar os fisioterapeutas no registro de dados funcionais, definição dos alvos de intervenção e documentação de desfechos, possibilitando a adoçâo de um novo modelo para orientar a prática clínica. Além de necessário sob o ponto de vista clínico e prático, essa forma de classificação é importante sob o ponto de vista econômico, uma vez que favorece a alocação de recursos compatíveis com as reais necessidades do indivíduo ${ }^{1,7}$.

O Acidente Vascular Cerebral (AVC) é a doença neurológica mais comum no mundo e tem sido cada vez mais frequente em adultos, tendo uma prevalência significativa em grupos etários jovens. É considerada a doença mais incapacitante, por trazer impacto em praticamente todas as funções humanas. Os principais desafios desses pacientes estão relacionados às incapacidades ou desvantagens, enquanto lidam com uma mudança de identidade, para uma pessoa incapacitada e o modo como isto afeta sua autoimagem e suas relações sociais. Sabendo que esses pacientes podem sofrer influências tanto de fatores contextuais (ambientais e pessoais) e seus impactos, tanto positivos quanto negativos, a utilização da CIF é de grande importância, lembrando que uma doença diagnosticada em diferentes indivíduos não causará o mesmo impacto $^{1,8,9}$. 
O termo AVC é usado para descrever sinais e sintomas neurológicos, usualmente focais e agudos que resultam de doença envolvendo vasos sanguíneos. O cérebro é muito susceptível a distúrbios do seu suprimento sanguíneo. Uma anóxia ou isquemia por somente alguns segundos podem causar sinais neurológicos e, em minutos dano neural irreversível. Embora a vascularização do cérebro possua características anatômicas e fisiológicas desenhadas para proteger o cérebro de comprometimentos circulatórios, quando esses mecanismos protetores falham o resultado é um $\mathrm{AVC}^{8}$.

$\mathrm{O}$ início do AVC é súbito, com déficit máximo no início, de modo que o choque para o paciente e seus familiares pode ser devastador ${ }^{10}$.

Existem dois tipos de AVC, podendo ser isquêmico o mais comum, que acontece devido à obstrução (trombose ou embolia) de uma das artérias cerebrais importantes, sendo a média a mais frequente, somando $70 \%$ dos casos ou, pode ser hemorrágico devido à hemorragia nas partes mais profundas do cérebro somando outros 20\% e $10 \%$ continuam inespecíficos. Ambos os tipos de AVC podem ocorrer em qualquer idade por muitas causas, incluindo doenças cardíacas, infecção, trauma, neoplasia, má formação vascular, desordens imunológicas, hipertensão arterial sistêmica (HAS), diabettes mellitus (DM), níveis altos de colesterol, obesidade, alto consumo de álcool, uso de cocaína e fumo. Quanto maior o número de fatores de risco presentes e/ou quanto mais elevado o grau de anormalidade de qualquer um dos fatores de risco, maior será o risco da ocorrência de um $\mathrm{AVC}^{10-13}$.

A consequência física mais comum após AVC é a hemiplegia, definida como "paralisia completa dos membros superiores e inferiores do mesmo lado do corpo". Outras sequelas podem ser problemas de percepção, cognição, sensoriais e de comunicação. Dependendo do grau de acometimento o indivíduo perde a seletividade dos seus movimentos, devido ao predomínio da atividade da musculatura antagonista, o que prejudica a realização das atividades de vida diária (AVD's) e consequentemente o seu retorno ao trabalho e ao convívio social ${ }^{10,13}$.

O local e o tamanho da lesão cerebrovascular e a quantidade de fluxo sanguíneo determinam o grau do déficit motor que pode se estender de uma incoordenação leve para uma paralisia completa de membros superiores e inferiores. Perdas motoras e sensoriais têm o maior efeito na mão, já que tendem a ter maior representação em ambos os hemisférios. As sensibilidades à dor, ao toque e à temperatura podem estar prejudicadas ou modificadas, mas normalmente não são perdidas. Pode ocorrer comprometimento grave da sensação de posiçáo da articulação, perda da discriminação entre dois pontos e da estereognosia, e dificuldade em reconhecer um estímulo tátil se um outro for simultaneamente realizado no lado intacto do corpo ${ }^{8}$.

Outros déficits pós-AVC podem ser categorizados como desordens da linguagem que também interferem na função cognitiva (disfasia que é caracterizada por perturbaçóes da linguagem); desordens perceptuais cognitivas (de percepção, orientação, memória, execução da função), depressão e outras perturbaçóes emocionais e comportamentais. Todos esses afetam o estado mental do indivíduo e têm potencial para afetar significativamente a habilidade do indivíduo ${ }^{8}$.

As consequências do AVC para o indivíduo são diversas e, geralmente permanecem por longos períodos, podendo atingir os dois níveis (Estrutura e Função do corpo, Atividade e Participação) do modelo de Classificaçấo Internacional de Funcionalidade (CIF) proposto pela Organização Mundial de Saúde (OMS) ${ }^{14}$.

Sabendo das sequelas deixadas pelo AVC é importante o uso da CIF pois, é uma classificação completa já que seus parâmetros permitem uma visão biopsicossocial do paciente. Essa classificação pode auxiliar na conduta a ser utilizada, tratando cada paciente de forma individual, realizando o tratamento com enfoque nas funçôes e restriçóes apresentadas pelos pacientes.

O objetivo geral deste trabalho consistiu em avaliar as funçóes e restrições de pacientes com sequela de AVC, utilizando como ferramenta a Escala de Classificação Internacional de Funcionalidade e Incapacidade e Saúde (CIF), incentivar o uso pelos profissionais da área da saúde e mostrar como a ferramenta permite uma visão maior das condiçôes de saúde dos pacientes, sabendo que estas condições podem ser influenciadas por outros fatores.

\section{MÉTODO}

\section{Amostra}

Primeiramente foi realizado o Estudo Piloto com 
duas pacientes que se encontravam em atendimento na Clínica Escola da Universidade Sáo Francisco (USF) que não foram selecionadas para fazer parte da coleta deste estudo, para observar possíveis dificuldades na aplicação das escalas.

Após a aprovação do Comitê de Ética e Pesquisa da USF, CAAF no 0011.0.142.000-09, os pacientes foram orientados a participar da pesquisa e assinaram o Termo de Consentimento Livre e Esclarecido.

\section{Procedimento}

Este estudo trata-se de relato de casos, onde foram avaliados cinco pacientes de ambos os gêneros, com quadro disfuncional de hemiparesia por sequela de AVC que se encontravam em atendimento fisioterapêutico na Clínica Escola da USF.

Inicialmente todos os pacientes foram avaliados pela ficha de avaliação neurológica utilizada na Clínica Escola, no qual possuem dados como: tipo de AVC, medicamentos em uso e exame físico. Os dados como: dados pessoais, profissão, doenças de base, lado acometido, queixa principal (QP), medicamentos em uso, outros tratamentos realizados, avaliação de tônus muscular, déficits sensorial e motor, marcha, coordenação motora, força muscular (FM) e Amplitude de Movimento (ADM) foram convertidos para os domínios Funçóes e Estruturas do Corpo, e Fatores Ambientais da CIF.

Após essa etapa, foi aplicada a Escala de Qualidade de Vida Específica para AVE (EQVE-AVE), no qual os dados sobre: energia, papéis sociais, humor, papéis familiares, autocuidado, memória/concentração, função de extremidade superior e trabalho/produtividade foram referentes aos Fatores Pessoais, Atividade e Participação da CIF.

A escala EQVE-AVE é um instrumento que incorpora questôes relacionadas as dimensôes da CIF. Tem doze domínios (energia, papel familiar, linguagem, mobilidade, humor, personalidade, autocuidado, papel social, raciocínio, função de membro superior, visão e trabalho/produtividade) elaborados a partir de entrevistas. Ao todo, os quarenta e nove itens são distribuídos dentro destes doze domínios. Com três possibilidades de reposta desenvolvidas em uma escala de escore de cinco a um: (1) quantidade de ajuda necessária para realizar tarefas espe- cíficas, indo de nenhuma ajuda a ajuda total; (2) quantidade de dificuldade experimentada quando é necessário realizar uma tarefa, indo de nenhuma dificuldade a incapaz de realizar a tarefa; (3) grau de concordância com afirmaçóes sobre sua funcionalidade, indo de discorda fortemente a concorda fortemente. $\mathrm{O}$ ponto de referência para todos os itens se refere à semana anterior ${ }^{9,15}$.

Por último foi aplicada a avaliação do Desempenho Ocupacional, empregando-se o Canadian Occupational Performance Measure (COPM). Os resultados referentes as AVD's, atividade produtiva e atividades de lazer, foram utilizados para mensurar os aspectos referentes à Atividade e Participação (CIF) desses pacientes.

A COPM é uma ferramenta que operacionaliza os principais conceitos do Modelo Canadense de Desempenho e das Diretrizes da Terapia Ocupacional para a Prática Baseada no Cliente. É um teste padronizado que tem como objetivo documentar a autopercepção do indivíduo sobre o impacto de uma condição de saúde, doença ou trauma no desempenho de suas tarefas funcionais. A perspectiva do cliente é obtida por meio de uma entrevista semiestruturada, no qual o paciente indica cinco tarefas funcionais que sejam mais significativas para ele e cujos desempenhos estejam comprometidos, nas AVD's (cuidados pessoais, mobilidade funcional, independência fora de casa), produtividade (dificuldade no trabalho, tarefas domésticas e cursos de curta duração) e lazer (recreação calma, recreação agitada e socialização). Em seguida, esse faz uma autoavaliação de seu desempenho e de sua satisfação com relação ao desempenho nas respectivas tarefas funcionais, sendo o escore total de dez pontos ${ }^{16-18}$.

\section{RESULTADOS}

A idade dos pacientes variou entre trinta e cinco anos e oitenta e cinco anos. Todos os pacientes apresentaram importantes restriçóes em suas AVD’s e também na participação social. A grande maioria deixou de realizar sua principal ocupação anterior ao AVC. Todos fazem uso de medicamentos sendo que dois possuem doenças de base como a HAS e DM e relataram que o tratamento Fisioterapêutico melhorou os sinais e sintomas deixados pelo AVC.

A paciente RFC de quarenta e cinco anos, sofreu um Acidente Vascular Cerebral do tipo Hemorrágico 
(AVCh) há quatro anos e tem como principal queixa o desequilíbrio e a instabilidade na marcha (Figura 1). Tem como doença de base a HAS e faz uso de medicamentos. Apresenta dificuldades nas tarefas domésticas como: na limpeza e na lavagem de roupas, devido ter dificuldades em realizar movimentos finos com as mãos. Refere também a sensação de cansaço durante o dia, tendo que parar sua tarefa e se sentar o que lhe causa grande impacto, visto que sua principal ocupação são as atividades domésticas. Quanto à mobilidade também apresenta grande dificuldade, pois faz uso de bengala e apresenta desequilíbrio durante a marcha e diminuição de $\mathrm{ADM}$ de Membro Inferior Direito (MID), o que dificulta seu deslocamento dentro e fora de casa tendo sua independência comprometida com restriçôes em sua participação social como o fato de ir a "missa" e impossibilidade de realizar atividades artesanais como pintura e crochê o que, anteriormente eram suas principais atividades de lazer, sendo assim sente-se desanimada quando pensa em seu futuro não tendo perspectiva de vida.
A paciente BFS de trinta e cinco anos, sofreu um Acidente Vascular Cerebral do tipo Isquêmico (AVCi) há quatro anos, tem como principal queixa a dificuldade de movimentar o Membro Superior Direito (Figura 2). Realiza tratamento psicológico, faz uso de medicamentos e tem sua mãe como cuidadora, o que é de extrema importância para ela, pois teve o AVC muito jovem e com isso teve que mudar radicalmente sua vida deixando seu emprego e sua casa em outra cidade e voltar a morar com a mãe. Apresenta afasia e dificuldade de concentração e memorização, o que a impossibilita de voltar à ocupação prévia ao AVC. Possui um déficit de sensibilidade em hemicorpo direito e apresenta dificuldades na marcha restringindo-a de realizar as atividades de lazer (cinema) sentindo-se insegura e pouco satisfeita com a vida.

O paciente CRM de oitenta e cinco anos sofreu um AVCi há aproximadamente cinco anos, tem como queixa principal a perda da marcha (Figura 3). Faz uso de medicamentos, utiliza cadeira de rodas para locomoção dentro e fora de casa, tem afasia de expressão, incoorde-

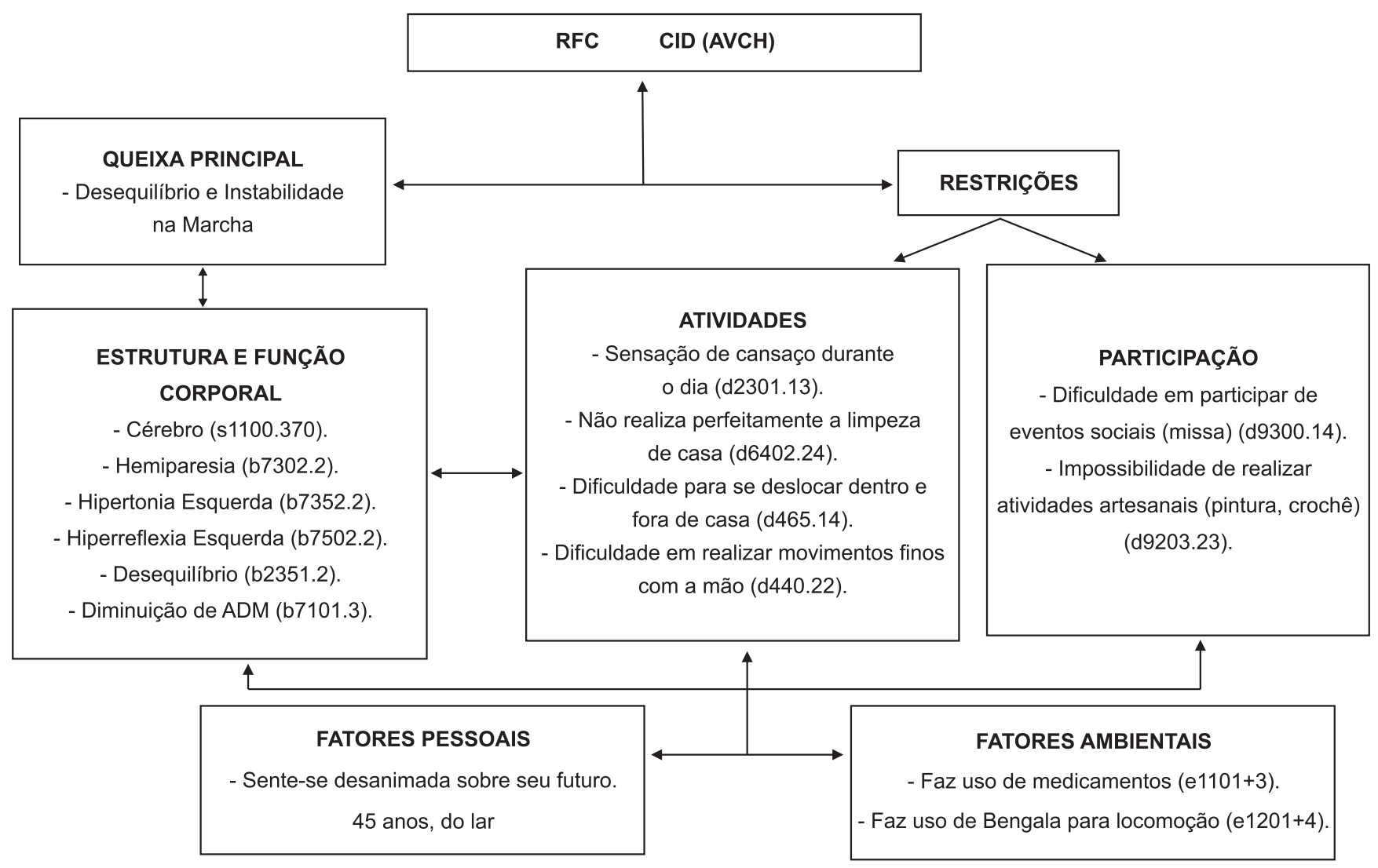

Figura 1. Caso Clínico 1. 


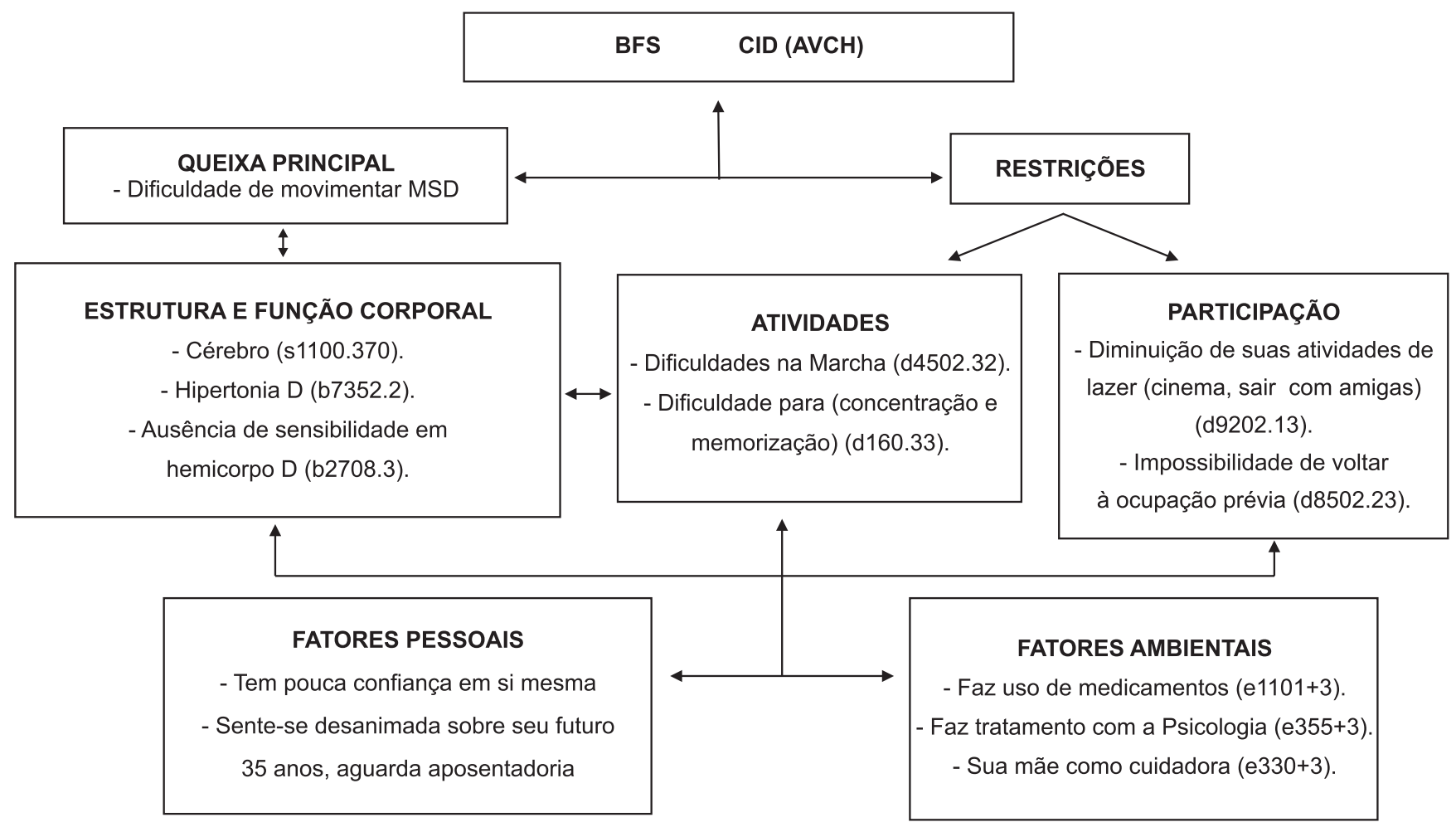

Figura 2. Caso Clínico 2.

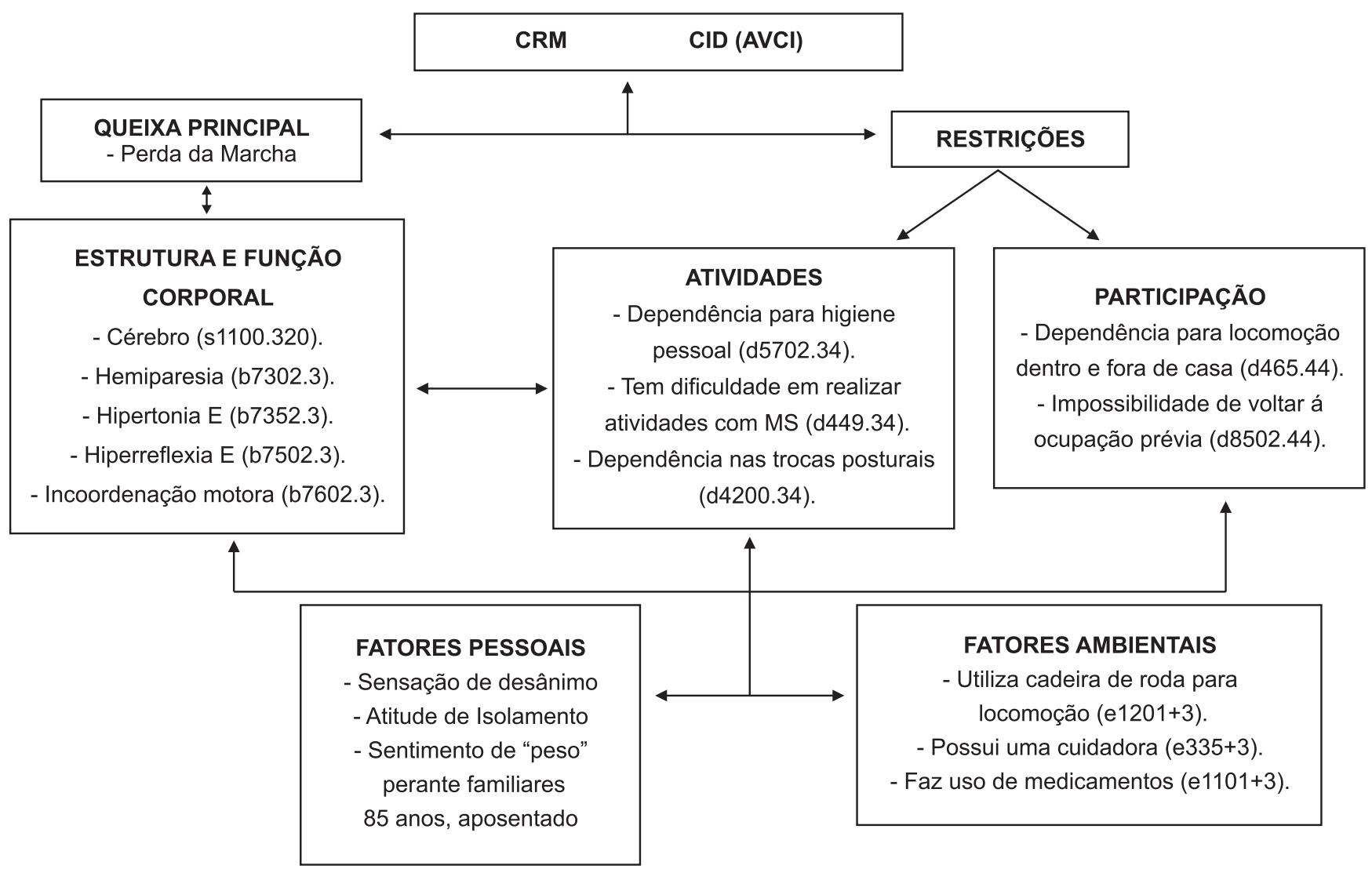

Figura 3. Caso Clínico 3. 


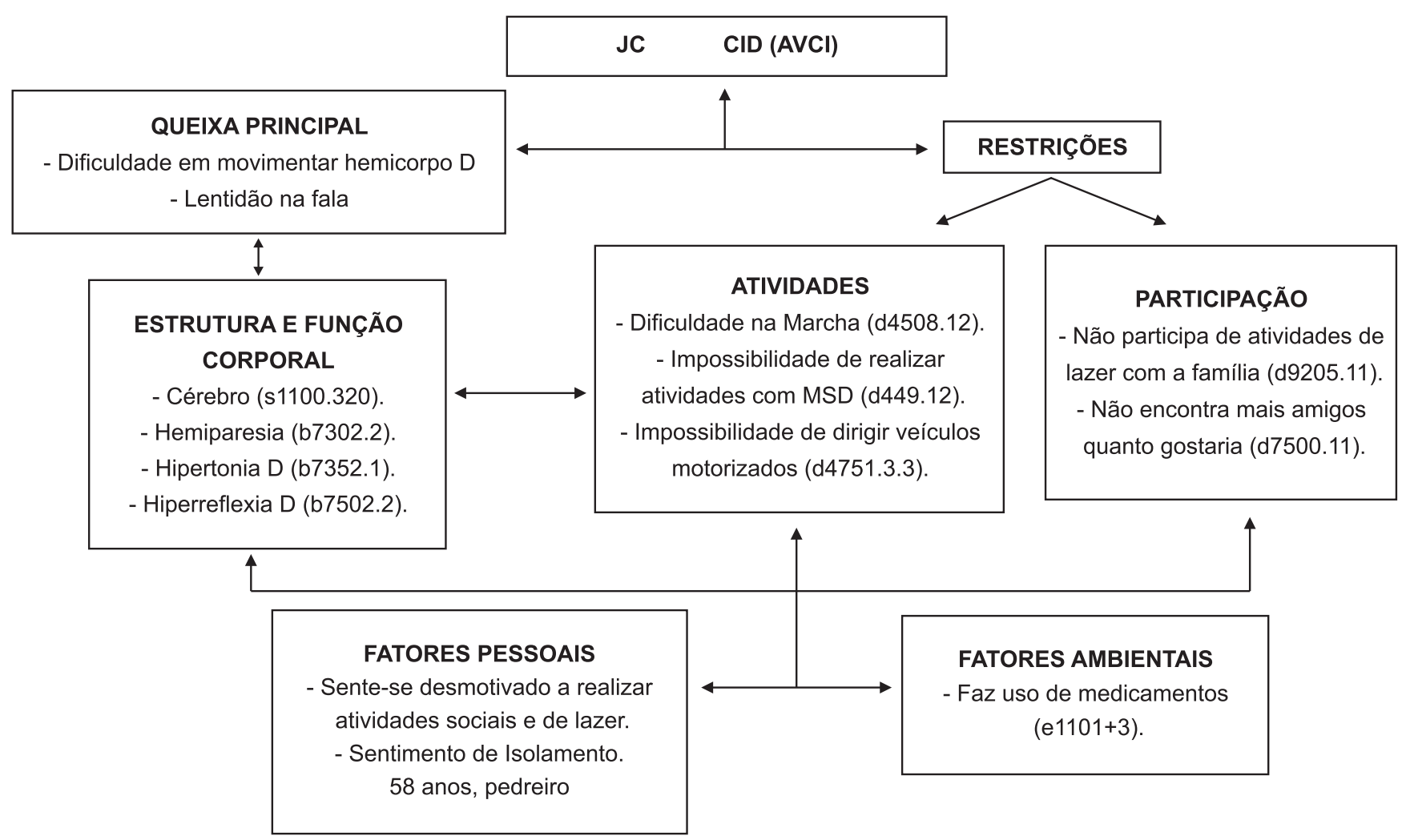

Figura 4. Caso Clínico 4.

nação motora, diminuição de FM e hipertonia do lado esquerdo do corpo, o que o torna dependente nas AVD's (higiene pessoal, trocas posturais). Todos esses aspectos o tornam incapaz de retornar a sua ocupação prévia. Sentese então desanimado e tem atitude de isolamento, além de se sentir como um "peso" perante seus familiares.

O paciente JC de cinquenta e oito anos sofreu um AVCi há aproximadamente um ano e sua queixa principal é a lentidão na fala e a dificuldade em movimentar o hemicorpo direito (Figura 4). Tem como doença de base a HAS e a DM e faz uso de medicamentos. Apresenta alteraçóes na mobilidade como fraqueza muscular em hemicorpo direito tendo entâo dificuldade na marcha. Também é impossibilitado de realizar tarefas complexas com o MSD como pentear cabelo, dirigir automóveis, o que acaba prejudicando o seu serviço de pedreiro e refletindo alteraçóes na participação social como sair com sua família nos horários de lazer ou então encontros com amigos. Com tudo isso fica desmotivado a realizar atividades sociais e de lazer com sua família e sente-se isolado por outras pessoas.
A paciente NO de sessenta e quatro anos, sofreu um AVCi há dois anos e tem como queixa principal dificuldade na marcha e fortes dores nas mãos (Figura 5). Faz tratamento com a Psicóloga e Psiquiatra, faz uso de medicamentos e utiliza muletas para locomoção. Apresenta incoordenação motora, diminuição de ADM, tem a sensação de cansaço durante a maior parte do dia, o que a faz ter dificuldades em permanecer em pé impossibilitando-a de realizar as atividades de lazer, as tarefas domésticas (limpar a casa, cozinhar) e de higiene pessoal, sendo necessário a ajuda total da cuidadora para realização das atividades. Tudo isso leva à impossibilidade de voltar a ocupação anterior ao AVC. Tem sentimento de desânimo e de "peso" perante a família e falta de paciência com os demais da família.

\section{DISCUSSÃO}

No presente estudo optou-se por aplicar a CIF em pacientes com diagnóstico clínico de AVC Isquêmico ou Hemorrágico, devido ao grande impacto que esta doença costuma causar na vida desses pacientes, afetando não 


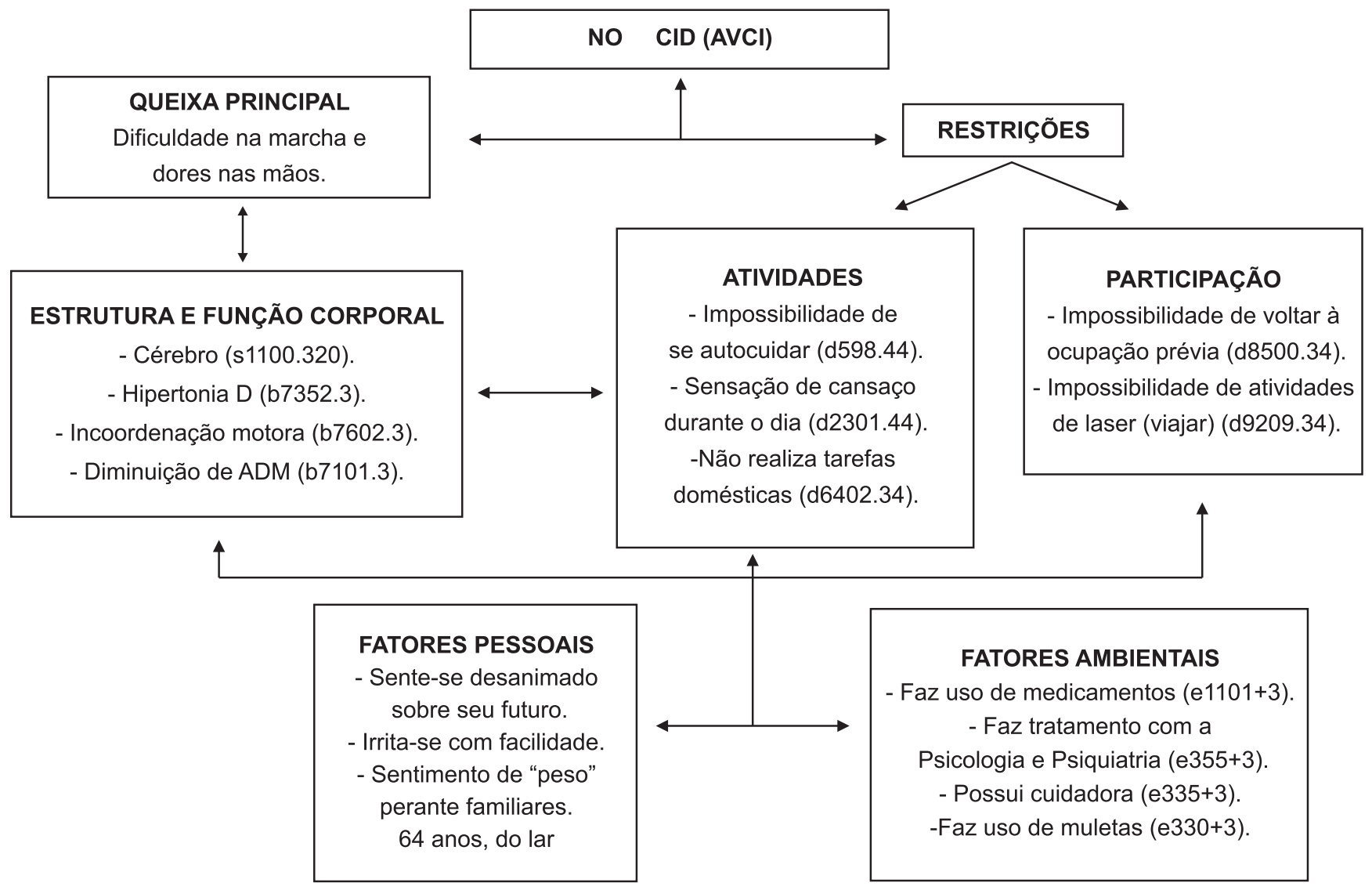

Figura 5. Caso Clínico 5.

somente eles, mas muitas vezes seus familiares, isso ocorrendo de forma particular, ou seja, pessoas com a mesma doença podem apresentar diferentes capacidades e limitaçôes, o que pode ser visualizado nos organogramas de 1 a 5. Além disso, com o uso da CIF conseguiu-se caracterizar o perfil funcional de cada paciente, baseando-se não apenas na estrutura e função corporal, mas em todas as dimensôes de saúde, incluindo as atividades e participação.

Ficou evidente neste estudo que, o paciente pósAVC sofre alteraçóes estruturais que o restringem em suas atividades diárias e em suas participações sociais. As alterações motoras como fraqueza muscular, espasticidade e padrões anormais de movimento, podem impedir ou dificultar as transferências, a deambulação e a realização de atividades básicas e instrumentais de vida diária, tornando o indivíduo fisicamente dependente, o que está de acordo com as queixas apresentadas pelos pacientes neste estudo, pois, três deles relataram problemas relacionados à marcha como, instabilidade e desequilíbrio. Outra queixa apontada por dois pacientes foi quanto à dificuldade em movimentar o membro superior. E, uma terceira queixa, foi referente aos problemas relacionados à fala, como afasia de expressão o que também é comum nesses pacientes de acordo com a literatura, no qual o paciente pode não ser capaz de falar ou entáo tem dificuldades em encontrar as palavras, mas é capaz de entender a fala dos outros. Portanto as incapacidades decorrentes do AVC afetam tanto a vida do paciente quanto a de seus familiares mais próximos, pois as medidas de reabilitação ocupam papel central no tratamento do paciente ${ }^{14,19,20}$.

A condição crônica de saúde traz experiências de perdas para o paciente, tais como: perdas nas relaçóes sociais, financeiras e na capacidade física. $\mathrm{Na}$ maioria dos pacientes acometidos por um AVC, a locomoção é uma das funçôes mais comprometidas, e a impossibilidade de andar e de subir escadas impede o acesso aos ambientes, o que acaba levando ao isolamento social, fato observado nos pacientes que participaram do estudo ${ }^{21,22}$.

$\mathrm{O}$ fato da maioria dos pacientes pesquisados possuir mais de um facilitador em sua vida (como ter uma 
cuidadora, fazer uso de medicamentos, utilizar auxílios para locomoção e realizar outros tipos de tratamentos) ou somente um, não interferiu quanto ao comprometimento emocional, já que todos apresentaram sinais de comprometimento emocional nos fatores pessoais. Isso se deve às restriçôes sociais e familiares, pois o isolamento social atinge os pacientes e os familiares e isso tem influência negativa sobre sua qualidade de vida ${ }^{21}$.

Outro ponto que foi comum entre três dos pacientes foi o fato de não conseguirem retornar a ocupação prévia ao AVC, sendo este um fator que favorece a doenças psicológicas do tipo depressão. Embora a depressão pós-AVC (DPAVC), apresente elevada prevalência ainda é pouco diagnosticada. Entre seus possíveis mecanismos fisiopatogênicos, tem dois principais: ser um fenômeno decorrente do impacto psicológico que o AVC causa no paciente (devido as incapacidades e limitaçóes que pioram muito suas relaçôes familiares e/ou sociais, atividades da vida diária e que, ainda, são responsáveis por um grande número de absenteísmos no trabalho) ou ser uma doença resultante de lesão neuronal em uma região específica do cérebro ${ }^{23}$.

Alguns pacientes ficaram restritos em suas profissões devido ao fato de apresentarem disfunções motoras, cognitivas e psicológicas, sendo um fator limitante em suas atividades profissionais. Este status profissional também é modificado pelo AVC, pois o paciente se desloca da condição de trabalhador para a de aposentado o que também gera grandes alteraçóes emocionais ao paciente?

As doenças e as incapacidades crônicas acabam gerando repercussóes sociais na saúde pública e na previdência social, pois pessoas incapacitadas geram custo para o governo do país. Vários estudos relatam que o AVC é a maior causa de incapacitação da população na faixa etária superior a cinquenta anos, sendo responsável por $10 \%$ do total de óbitos, 32,6\% das mortes com causas vasculares e $40 \%$ das aposentadorias precoces no Brasil. Um paciente com AVC custa para o Sistema Único de Saúde (SUS), em média, R\$ 6 mil, mas esse valor varia de acordo com a gravidade de cada caso ${ }^{21,24}$.

De acordo com o estudo realizado e com várias literaturas fica evidente a importância do uso da CIF, para avaliarmos os pacientes não somente de acordo com seu diagnóstico clínico e sim como um todo ${ }^{7,24,25}$.
Neste estudo todos possuíam o mesmo diagnóstico clínico, mas se apresentaram com diferentes disfunções, isso nos mostra que podemos planejar condutas específicas para cada paciente trabalhando de forma individual.

O modo como a CIF foi estruturada, com seus componentes que abrangem várias condiçóes de saúde faz com que seja capaz de auxiliar todos os profissionais a enxergarem o paciente de forma individual, podendo evidenciar fatores preocupantes e/ou situaçôes que não são vistas em uma simples avaliação periódica, mas que acabam interferindo em sua vida e em sua participação social.

A CIF por ser uma ferramenta que abrange várias condições fazendo com que seu uso seja interessante tanto na área da saúde como na política no serviço público pois, se usada por vários profissionais em diferentes países, pode levar a uma linguagem unificada gerando comparaçóes entre grupos de saúde e entre países.

Dessa forma, além de necessário para o ponto de vista clínico e prático, esse novo modelo de classificação é importante sob o ponto de vista econômico, uma vez que favorece a alocação de recursos compatíveis com as reais necessidades do serviço ${ }^{7}$.

\section{CONCLUSÃO}

O presente estudo evidenciou que a CIF possui parâmetros adequados para a avaliação dos pacientes com sequela de AVC, já que permite uma visão global, abrangendo desde a estrutura corporal comprometida até os fatores pessoais e ambientais. Destarte, a funcionalidade do paciente passa a ser um componente de saúde e não apenas uma consequência da doença.

\section{REFERÊNCIAS}

1.Sampaio FR, Mancini MC, Gonçalves GGP, Bittencourt NFN, Miranda AD, Fonseca ST. Aplicação da Classificação Internacional de Funcionalidade, Incapacidade e Saúde (CIF) na prática clínica do Fisioterapeuta. Rev Bras de Fisioter 2005;9:129-36.

2.Norma F, Buchalla MC. A Classificação Internacional de Funcionalidade, Incapacidade e Saúde da Organização Mundial da Saúde: conceitos, usos e perspectivas. Rev Bras de Epidemiol 2005;8:93-187.

3.Di Nubila VBH. Aplicação das Classificações CID-10 e CIF nas definições de deficiência e incapacidade (Tese). São Paulo: Faculdade de Saúde Pública da 
Universidade de São Paulo; 2007. 181p. Doutorado em saúde pública. 4.Organização Mundial da Saúde (OMS). CIF - Classificação Internacional de Funcionalidade, Incapacidade e Saúde. São Paulo, Edusp, 2008, 325p.

5.Sampaio FR, Luz TM. Funcionalidade e Incapacidade humana: explorando um escopo da Classificação Internacional da Organização Mundial da Saúde. Cad. de Saúde Pública 2009;25:475-83. http://dx.doi.org/10.1590/S0102-311X2009000300002

6.Organização Mundial da Saúde (OMS). Classificaçăo Internacional de Funcionalidade Incapacidade e Saúde. 2003. [Acessado em: 22 out. 2008]; Disponível em: http://arquivo.ese.ips.pt/ese/cursos/edespecial/CIFIS.pdf.

7.Sabino SG, Coelho MC, Sampaio FR. Utilização da Classificação Internacional de Funcionalidade, Incapacidade e Saúde na Avaliação Fisioterapêutica de indivíduos com problemas musculoesqueléticos nos membros inferiores e regiăo lombar. Acta Fisiatr 2008;15:24-30.

8.Carr HJ, Shepherd BR. Acidente Vascular Cerebral. In: Reabilitação Neurológica: Otimizando o Desempenho Motor. Sáo Paulo: Manole; 2008, p.253-73. 9.Neto G. Aplicação da Escala de Qualidade de Vida Específica para AVE (EQVE-AVE) em Hemiplégicos Agudos: Propriedades Psicométricas e sua Correlação com a Classificação Internacional de Funcionalidade, Incapacidade e Saúde. [Dissertação]. Belo Horizonte: Universidade Federal de Minas Gerais; 2007, 77p. Mestrado.

10.Durward B, Baer G, Wade J. Acidente Vascular Cerebral. In: Stokes. M. Neurologia para Fisioterapeutas. São Paulo: Premier; 2000, p.83-99.

11.Ryerson DS. Hemiplegia Resultante de Dano ou Doença Vascular. In: Umphred, A.D. Fisioterapia Neurológica. São Paulo, Manole; 1994, p.615-55.

12.Polese CJ, Tonial A, Jung KF, Mazuco R, Oliveira GS, Schuster CR. Avaliaçấo da Funcionalidade de indivíduos acometidos por Acidente Vascular Encefálico. Rev Neurocienc 2008;16:175-8.

13.Araújo SPA, Silva FCP, Moreira SPCR, Bonilha FS. Prevalência dos Fatores de Risco em pacientes com Acidente Vascular Encefálico atendidos no setor de Neurologia da Clinica de Fisioterapia da Unipar-Campus Sede. Arq. Ciência Saúde Unipar 2008;12:35-42.

14.Teixeira-Salmela LF, Faria CDCM, Guimarăes CQ, Goulart F, Parreira VF, Inácio EP, et al. Treinamento Físico e Destreinamento em Hemiplégicos Crônicos: Impacto na Qualidade de Vida. Rev Bras Fisioter 2005;9:347-53.

15.Lima MCR. Adaptação Transcultural do Stroke Specific Quality of Life -
SSQOL: Um instrumento específico para avaliar a qualidade de vida de hemiplégicos. [dissertaçáo]. Belo Horizonte: Universidade Federal de Minas Gerais; 2006, 77p. Mestre em Ciências da Reabilitação.

16.Cabral AHL. Fatores Associados ao Retorno ao Trabalho após um trauma de mão: uma abordagem qualiquantitativa. [Dissertação]. Belo Horizonte: Universidade Federal de Minas Gerais; 2007, 39p. Mestrado.

17.Figueiredo IM, Sampaio RF, Mancini MC, Nascimento MC. Ganhos Funcionais e sua relação com os componentes de função em trabalhadores com Lesão de Mão. Rev Bras Fisioter 2006;10:421-7.

18.Garros CSD. Avaliação do Desempenho e da Satisfaçấo Pessoal do Paciente com Mão Espástica após uso da Órtese Dorsal Volar. [Dissertação]. São Paulo: Faculdade de Ciências Médicas da Santa Casa de São Paulo; 2008, 72p. Mestrado.

19.Magalhães AL, Bilton LT. Avaliação de Linguagem e de deglutição de pacientes hospitalizados após Acidente Vascular Cerebral. Distúrbios da Comunicação. 2004;16:65-81.

20.Andrade OMP, Ferreira OF, Haase GV. O uso da CIF através do trabalho interdisciplinar no AVC pediátrico: relato de caso. Contextos Clínicos 2009;2:27-39.

21.Brito SE, Rabinovich PE. A família também adoece! Mudanças secundárias à ocorrência de Acidente Vascular Encefálico na família. Interface 2008;12:783-94.

22.Segura ACD, Brushi AF, Golin BT, Gregol F, Bianchini MK, Rocha P. A evolução da marcha através de uma conduta cinesioterapêutica em pacientes hemiparéticos com sequela de AVE. Arq Ciência Saúde Unipar 2008;12:25-33. 23.Ribeiro SD, Filho CAE, Losapio FM, Sena PE. Terapia medicamentosa na depressão pós acidente vascular encefálico. J.bras.psiquiatr. 2009;58:135-142. 24.Abramczuk B, Villela E. A luta contra o AVC no Brasil. Com Ciência: Revista Eletrônica de Jornalismo Científico [internet]. 2010 [acessado em: 01 out. 2010]. Disponível em: http://www.comciencia.br/comciencia/handler. php? section $=8 \&$ edicao $=47 \&$ id $=582$.

25.Andrade ML, Costa MFM, Caetano AJ, Soares E, Beserra PE. A problemática do cuidador familiar do portador de Acidente Vascular Cerebral. Rev Esc Enferm USP 2008;43:37-43.

26.Sampaio RF, Mancini MC. Tecendo uma rede de usuários da CIF. Rev Bras Fisioter 2007;11:5-6. 